\title{
THE INTERSTELLAR DUST EMISSION SEEN BY ISO
}

\author{
J.L. PUGET \\ Institut d'Astrophysique Spatiale \\ Université Paris Sud \\ Orsay, France
}

\section{Confirmation of the three component model of interstellar dust}

Among the important contributions of ISO to the physics of the interstellar medium, this paper concentrates only on the emission from the solid phase (the interstellar grains) and the small atom clusters which are the bridge between the grains and the small molecules with less than about 10 atoms.

The transiently heated very small particles model including polycyclic aromatic hydrocarbons (PAHs) was introduced 15 years ago (see Puget, Leger 1989 for a review) to explain the unidentified infrared bands and the strong photometric emission detected by IRAS at 12 and $25 \mu \mathrm{m}$ everywhere in the galaxy, including cirrus clouds heated by the general interstellar radiation field (ISRF). The model could not be properly tested before ISO with its cryogenically-cooled payload giving unprecedented sensitivity to low brightness extended sources. Now ISO has brought a striking confirmation of the two major predictions of this model.

The emission in the mid-infrared IRAS photometric bands from cirrus clouds was interpreted as due to the vibration bands of PAH molecules (neutral and ionized) containing $15 \%$ of all cosmic carbon and absorbing about $30 \%$ of the interstellar radiation (mostly soft UV and visible). ISOPHOT data (Laureijs et al. 1996) showed that the relative strength of specific narrow filters centered on the vibration bands of PAHs confirmed the presence of the expected bands in the diffuse galactic emission already seen in a balloon experiment ( $3.3 \mu \mathrm{m}$ (Giard et al 1988) and $6.2 \mu \mathrm{m}$ (Giard et al 1994)). ISOPHOT-S observations by Mattila et al (1996) found no evidence for a continuum below the features. A Circular Variable Filter spectrum obtained with ISOCAM by Boulanger et al. (1997) showed all the expected features including broad underlying continuum rising at $\lambda>? 6 ? \mu \mathrm{m}$ and falling at the longest wavelengths accessible with ISOCAM $(\lambda<16 \mu \mathrm{m})$.

The second strong prediction of the out-of-equilibrium emission is that, when the exciting radiation field increases by several orders of magnitude, the intensity should increase proportionally but the spectrum should stay the same. This is qualitatively different from what happens at longer wavelengths where the big grains radiate at their equilibrium temperature: the temperature of the emission changes when the intensity of the radiation changes. Bernard et al 1997 compare the CVF spectra of a cirrus cloud in Chameleon heated by the ISRF with a spectrum taken on the edge of the Rho Ophiucus molecular cloud heated by nearby B stars (about 25 times the ISRF) and a third spectrum taken in the reflection nebula NGC2023 (exciting field about a thousand times the ISRF). The remarkable result is that the three spectra, after scaling by factors 25 and 1000 , are identical within a few per cent!

The "constant spectrum" is predicted to breakdown when the radiation spectrum becomes strong enough to photothermodissociate or doubly ionise and Coulomb dissociate the PAHs. Then the size spectrum of interstellar particles changes and the spectrum changes.

Using the SWS instrument in the full spectrum grating mode, Roelfsema et al.(1996) on a set of compact HII regions and Verstraete et al. (1996) on a series of 10 spectra across the ionizing front of M 17 have shown that the spectrum starts to change abruptly when the uv radiation field becomes larger than $10^{5}$ times the ISRF. The 6.2, 7.7 and 8.6 narrow features are replaced by a broad feature between 6 and $10 \mu \mathrm{m}$ peaking at about $8.5 \mu \mathrm{m}$. 
A similar transition is seen in galactic nuciei where the dust is heated by a hard, intense spectrum. On bright photodissociation regions or reflection nebulae when the narrow features still dominate, the SWS spectrometer allows the attainment of high resolution high signal-to-noise spectra.

A very interesting characteristic of the spectra is that they are very smooth even in the wings of the narrow features. In the Leger and Puget (1984) model, the features are made of the contribution of many narrower lines observed in the laboratory and slightly displaced for each individual molecule (sce for example the resulting spectrum for a laboratory mixture of about 50 molecules, Moutou 1996). The lower intensity in the wings results from a decreasing density of lines and thus the spectrum should show an increasing relative structure in the wings. To account for the total absence of structure the number of individual molecules should be large to account for the smoothness of the narrow features.

Boulanger et al. (1997) have shown that the profiles can be fitted very well by Lorentzian ones; although one cannot rule out the possibility of a very large number of species contributing more or less equally to the features, these two elements are strongly suggestive of a broadening mechanism at work.

It is known that the PAH molecules observed in the laboratory are smaller than the ones expected to dominate in the interstellar medium.

An interstellar aromatic cluster of 300 carbon atoms is not necessarily a single planar aromatic molecule. It could be made of a dozen PAH molecules (made of about 25 carbon atoms each) loosely bound, and containing a fraction of saturated hydrocarbons. This is what happens in coals which are known to have absorption spectra very similar to the interstellar emission spectra (Guillois et al 1994).

Thus an interesting question raised by the ISO data is the following: Are the PAH molecules of the interstellar medium isolated or in small clusters? It should be noted that this does not change the basic assumption of the 3 component model (Desert et al. 1990, Dwek 1997): aromatic molecules being the basic component of the smallest interstellar atom clusters, small enough to radiate after single photon excitation.

ISO data also open the door to the identification of the intermediate size component. The spectra of highly excited region where the smaller particles are destroyed and where they dominate should lead to some clue about their chemical nature.

On the third component of the model (the big grains) ISO is also bringing new results. The same crystalline silicate have been seen in an almost pure form both in the Hale Bopp Comet and in oxygen rich stellar envelopes.

\section{Interstellar ices}

It is well known that in dense regions of molecular clouds and particularly in protostellar cores, small molecules like $\mathrm{CO}, \mathrm{H}_{2} \mathrm{O}$, condense on to cold grains. The ISO SWS has given high resolution, high signal to noise full spectra of embedded sources (Whittet et al 1996, d'Hendecourt et al 1996,).

Laboratory mixture of ices $\left(\mathrm{H}_{2} \mathrm{O}, \mathrm{CO}, \mathrm{CH}_{4}, \mathrm{NH}_{3}, \mathrm{O}_{2}\right)$ after photolysis show most of the features seen in the interstellar spectra. Nevertheless the 6.8 micron feature remains unexplained.

The complete ISO spectra have allowed to rule out several of the explanations previously proposed (alcohols, $\mathrm{NH}_{4}+$, carbonates) because predicted features in parts of the spectrum not observed before ISO where not detected. This illustrates well the power of ISO for this problem.

These spectra confirm the presence of large quantities of condensed $\mathrm{CO}_{2}$ on most lines of sight (de Graalıw et al 1996, van Dishoeck 1996) seen at $4.6 \mu \mathrm{m}$ for the first time. An interesting result is the very different ratio of gas to solid phase observed for $\mathrm{CO}_{2}$ and $\mathrm{H}_{2} \mathrm{O}$ (see also de Graauw and Cernicharo, this volume).

Solid methane was also detected (Boogert et al 1996). The quality of the spectra allows the testing of methane mixed with polar or non polar ice and an estimation of the temperature. The temperature seems to be low $(10 \mathrm{~K})$ along the line of sight observed.

\section{References}

Bernard et al 1997, in" Diffuse infrared radiation and the IRTS", ASP conf 124,161

Boogert ct al, A. and A. Letters 315, L377 
Boulanger et al. 1997, A. and A to be published

de Graaw et al, A. and A. Letters 315, L345

Desert F. X., Boulanger F., and Puget J.L. 1990, A. and A 237, 215

d'Hendecourt et al 1996, A. and A. Letters 315, L365

Dwek 1997, Ap. J. in press

Giard et al 1988, A. and A. Letters 201, L1

Giard et al 1994, A. and A. 286, 203

Guillois O., Nenner L., Papoular R. and Reynaud C. 1994, A. and A. 285, 1003

Helmich ert al, A. and A. Letters 315, L173

Laureijs et al, 1996, A. and A. Letters, 315, L?313

Leger A and Puget J. L., 1984, A. and A. Letters 137, L5

Mattila et al 1996, A. and A. Letters 315, L353

Moutou 1996, $\mathrm{PhD}$ thesis, Paris

Puget J. L. and Leger A., 1989, Annual Review of Astron. and Ap. 27,161

Roelfsema et al.1996 A. and A. Letters, 315, L289

Ryter et al 1997 , in preparation

Schutte et al, A. and A. Letters 315, L333

van Dishoek et al, A. and A. Letters 315, L177

Verstraete et al. 1996, A. and A. Letters, 315, L337

Whittet et al , A. and A. Letters 315, L357 